\title{
Recrutamento alveolar com suspiro: impacto na mecânica respiratória e oxigenação de pacientes ventilados mecanicamente
}

\author{
Alveolar recruitment: impact on respiratory \\ mechanics and oxygenation of mechanically \\ ventilated patients
}

\author{
Artur Ferreira Batista Neto' ${ }^{1}$ \\ Katia de Miranda Avena ${ }^{2}$ (1) \\ Kristine Menezes Barberino Mendes ${ }^{3}$ (1)
}

Helder Brito Duarte 4

Flávia Milholo Olivieri ${ }^{5}$ (1) Luciana Ferreira Feijó ${ }^{6}$ (C)

1Autor para correspondência. Hospital da Cidade, Secretaria da Saúde do Estado da Bahia (Salvador). Bahia, Brasil. afbn5@yahoo.com.br
2.6 Hospital da Cidade, Secretaria da Saúde do Estado da Bahia (Salvador). Bahia, Brasil. katiaavena@hotmail.com, kbarberino@gmail.com,
helderphysio@gmail.com, olivierifau@gmail.com, lucianafeijo@gmail.com

RESUMO | INTRODUÇão: O suspiro caracteriza-se pela realização de uma inspiração lenta e profunda, seguida de uma expiração lenta. Estudos sugerem que a adição de um suspiro por minuto em pacientes com síndrome do desconforto respiratório agudo, ventilados em PSV, melhora a oxigenação e a mecânica pulmonar. OBJETIVO: Avaliar o impacto da manobra de recrutamento alveolar através de suspiro na mecânica pulmonar e oxigenação em pacientes ventilados mecanicamente, além de verificar o impacto hemodinâmico e a incidência de intercorrências associadas à utilização da técnica. MATERIAIS E MÉTODOS: Estudo experimental com 17 pacientes em ventilação mecânica, apresentando relação entre pressão parcial de oxigênio alveolar e fração inspirada de oxigênio $\left(\mathrm{PaO}_{2} / \mathrm{FiO}_{2}\right)$ inferior a $300 \mathrm{mmHg}$. Avaliou-se dados respiratórios, de mecânica pulmonar e hemodinâmicos. Os dados foram coletados durante três períodos: antes do suspiro, imediatamente após e 15 minutos depois da técnica. Dois suspiros por minuto foram administrados utilizando pressão em vias aéreas limitada em $40 \mathrm{cmH}_{2} \mathrm{O}$, durante um tempo inspiratório de quatro segundos. RESULTADOS: Após o suspiro, observou-se aumento da $\mathrm{PaO}_{2^{\prime}}$ pressão resistiva, complacência estática e relação $\mathrm{PaO}_{2} / \mathrm{FiO}_{2^{\prime}}$ além de diminuição da pressão de platô e pressão parcial de gás carbônico alveolar $\left(\mathrm{PaCO}_{2}\right)$. Após 15 minutos da retirada do suspiro observou-se que a $\mathrm{PaO}_{2^{\prime}}$ pressão resistiva, complacência estática e relação $\mathrm{PaO}_{2}$ ' $\mathrm{FiO}_{2}$ mantiveram-se acima do valor basal, enquanto que a pressão de platô manteve-se abaixo. Não foi observada alteração significante nas variáveis hemodinâmicas. CONCLUSÃO: $\mathrm{O}$ suspiro em pacientes ventilados mecanicamente foi capaz de melhorar a oxigenação e a mecânica pulmonar sem comprometer a estabilidade hemodinâmica.

PALAVRAS-CHAVE: Síndrome do Desconforto Respiratório Agudo. Ventilação Mecânica. Modalidades de Fisioterapia. Mecânica Respiratória. Oxigenação.
ABSTRACT | INTRODUCTION: The sigh is characterized by a slow and deep inhalation, followed by a slow exhalation. Studies suggest that the addition of one breath per minute in patients with acute respiratory distress syndrome, ventilated on PSV, improves oxygenation and pulmonary mechanics. OBJECTIVE: Analyze the impact of the alveolar recruitment maneuver through breath in pulmonary mechanics and oxygenation in mechanically ventilated patients, in addition to checking the hemodynamic impact and the incidence of complications associated with the use of the technique. MATERIALS AND METHODS: Experimental study with 17 patients on mechanical ventilation, showing a relationship between partial pressure of alveolar oxygen and fraction of inspired oxygen $\left(\mathrm{PaO}_{2} / \mathrm{FiO}_{2}\right)$ below $300 \mathrm{mmHg}$. Respiratory, pulmonary mechanics, and hemodynamic data were evaluated. Data were collected during three periods: before sigh, immediately after, and 15 minutes after the technique. Two sighs per minute were administered using airways pressure limited to $40 \mathrm{cmH}_{2} \mathrm{O}$, during an inspiratory time of four seconds. RESULTS: After the sigh, there was an increase in $\mathrm{PaO}_{2}$, resistive pressure, static compliance, and $\mathrm{PaO}_{2} / \mathrm{FiO}_{2}$ ratio, in addition to a decrease in plateau pressure and partial pressure of alveolar carbon dioxide $\left(\mathrm{PaCO}_{2}\right)$. After 15 minutes of sigh removal, it was observed that $\mathrm{PaO}_{2}$, resistive pressure, static compliance, and $\mathrm{PaO}_{2}$ / $\mathrm{FiO}_{2}$ ratio remained above the baseline, while the plateau pressure remained below. There was no significant change in hemodynamic variables. CONCLUSION: The sigh in mechanically ventilated patients was able to improve oxygenation and pulmonary mechanics without compromising hemodynamic stability.

KEYWORDS: Respiratory Distress Syndrome. Adult. Respiration. Artificial. Physical Therapy Modalities. Respiratory Mechanics. Oxygenation. 


\section{Introdução}

A evolução da ciência está alinhada à melhoria da sobrevida dos pacientes na Unidade de Terapia Intensiva (UTI), onde observa-se um progressivo aperfeiçoamento tecnológico na assistência prestada. A compreensão quanto ao custo biológico imposto pelo suporte orgânico de vida direciona para o surgimento de novas estratégias terapêuticas. Nos últimos 50 anos, no âmbito do tratamento da síndrome do desconforto respiratório agudo (SDRA) e da lesão pulmonar aguda (LPA), a ventilação mecânica invasiva (VM) tem evoluído tanto do ponto de vista tecnológico, quanto fisiológico e terapêutico¹.

A SDRA e LPA produzem impacto direto na mecânica ventilatória, atingindo alvéolos e interstício, com redução da produção de surfactante e infiltração de células inflamatórias 2 . Neste contexto, a VM deve ser aplicada de forma estratégica, eliminando parâmetros prejudiciais que possam elevar os danos causados não somente aos pulmões, mas também a todo o sistema. Dessa forma, a estratégia de ventilação protetora é indicada no intuito de prevenir sobredistensões alveolares (barotrauma/volutrauma), lesão por cisalhamento com insuflação e desinsuflação e/ ou proliferação inflamatória sistêmica (biotrauma) nas áreas saudáveis (baby lung) ${ }^{3}$.

O conceito de ventilação protetora vem sendo aprimorado constantemente e consiste em utilizar volume corrente em $6 \mathrm{~mL} / \mathrm{Kg}$ do peso ideal estimado pela altura, pressão de platô (PPLATÔ) menor que $30 \mathrm{cmH}_{2} \mathrm{O}$ com pressão positiva no final da expiração (PEEP) a ser ajustada com base na mecânica ventilatória via Complacência Estática do Sistema Respiratório $\left(\right.$ Cest $_{\mathrm{s}, \mathrm{r}}$ ) ou guiado pela tabela de baixa PEEP $\times \mathrm{FiO}_{2}$ (Fração Inspirada de Oxigênio) - tendo como parâmetro uma saturação periférica de oxigênio superior a $92 \%$. A frequência respiratória pode ser ajustada até 35ipm (desde que não ocasione auto PEEP), de acordo com a pressão parcial de gás carbônico alveolar $\left(\mathrm{PaCO}_{2}\right)$ desejada, com uma determinada tolerância à hipercapnia ${ }^{4,5}$.
Contudo, a aplicação de baixo volume corrente pode favorecer um colapso das vias áreas dependentes e diminuição da exalação de gás resultando em hipercapnia. Sendo assim, a estratégia ventilatória de suspiro pode ser indicada ${ }^{6,7}$.

O suspiro fisiológico caracteriza-se pela realização de uma inspiração lenta e profunda, seguida de uma expiração também lenta. Ocorre de forma frequente e irregular em indivíduos saudáveis. Portanto, assim como qualquer inspiração profunda, tem a capacidade de elevar temporariamente a pressão parcial de oxigênio alveolar $\left(\mathrm{PaO}_{2}\right)$, diminuir a $\mathrm{PaCO}_{2}$ e aumentar o retorno venoso ao coração?

A manobra de recrutamento alveolar através de suspiro é considerada por alguns autores como "mais fisiológica" 9 . O papel do suspiro durante a VM em pacientes com SDRA tem sido enfatizado e a entrega automática e periódica desses suspiros por um ventilador pode ser considerada como um possível componente da estratégia ventilatória nestes pacientes ${ }^{10}$. É possível que estratégias ventilatórias que utilizem um tempo de suspiro mais prolongado possam favorecer o maior preenchimento das unidades alveolares através do melhor equilíbrio na redistribuição gasosa e das constantes de tempo do sistema respiratório ${ }^{11,12}$.

Existem evidências que a adição de um suspiro por minuto em pacientes com SDRA, ventilados em PSV, seja capaz de melhorar a oxigenação e a mecânica pulmonar, associado com a redução do drive respiratório ${ }^{10}$. Nesse contexto, a manobra de recrutamento alveolar através de suspiro promove benefícios como a diminuição da distensão regional, a melhora da oxigenação sem hiperdistensão adicional e a redistribuição da perfusão sem prejuízos ao tecido pulmonar através de eventos adversos (barotrauma, pneumotórax e piora da inflamação pulmonar) (13-17. $^{6}$.

Frente ao exposto, o objetivo desse estudo é avaliar o impacto da manobra de recrutamento alveolar através de suspiro na mecânica pulmonar e oxigenação em pacientes ventilados mecanicamente, além de verificar o impacto hemodinâmico e a incidência de intercorrências associadas à utilização da técnica. 


\section{Métodos}

Trata-se de um estudo experimental, não controlado, do tipo antes e depois, com caráter descritivo e analítico, realizado na Unidade de Cuidados Intensivos (UCl) de um hospital geral, de grande porte e alta complexidade, da rede pública do estado da Bahia, localizado na cidade de Salvador, Bahia, no período de dezembro/2009 a fevereiro/2010.

Foi utilizada uma amostragem não probabilística, por conveniência, onde foram incluídos pacientes intubados, de ambos os sexos, maiores de 18 anos, capazes de assistirem à prótese ventilatória, apresentando relação $\mathrm{PaO}_{2} / \mathrm{FiO}_{2}$ inferior a $300 \mathrm{mmHg}$ e que os responsáveis legais tivessem assinado o Termo de Consentimento Livre e Esclarecido (TCLE) consentindo a participação no estudo.

Foram excluídos do estudo os pacientes que com instabilidade hemodinâmica mesmo em uso de aminas vasoativas; portadores de alguma doença pulmonar prévia (como enfisema pulmonar e pneumotórax não tratado); e os traqueostomizados. O desenvolvimento de instabilidade hemodinâmica durante aplicação do protocolo (caracterizado pela PAM $<60 \mathrm{mmHg}$ ), depressão do drive respiratório e exteriorização de secreção traqueal foram considerados como critérios para interrupção da participação do paciente no estudo. Entretanto, estes não foram apresentados por nenhum dos pacientes incluídos no estudo.

Os dados sociodemográficos e clínicos coletados foram: idade (em anos), sexo (categorizado em masculino e feminino), índice de massa corpórea (IMC) em Kg/ $\mathrm{m} 2$, diagnóstico admissional, comorbidades hipertensão (HAS), diabetes mellitus (DM), tabagismo, alcoolismo, obesidade, pneumopatia, doença cardiovascular, neuropatias e neoplasias, e o fator que motivou à VM.

Os dados de mecânica pulmonar coletados foram: pressão de pico (PPICO), PPLATÔ, Pressão Resistiva (PR), Complacência Estática do Sistema Respiratório $\left(\mathrm{Cest}_{\mathrm{s}, \mathrm{r}}\right)$, pressão parcial de oxigênio arterial $\left(\mathrm{PaO}_{2}\right)$, pressão parcial de dióxido de carbono arterial $\left(\mathrm{PaCO}_{2}\right)$, relação $\mathrm{PaO}_{2} / \mathrm{FiO}_{2}$ e volume minuto (VE). A PR foi calculada pela diferença entre a pressão traqueal e a PPLATÔ, por meio de oclusão inspiratória de seis segundos, conforme a configuração do equipamento. Já a Cest $_{s, r}$ foi calculada pela razão entre volume corrente e diferença entre a PPLATÔ e a PEEP total.
Os dados hemodinâmicos coletados foram frequência cardíaca (FC), pressão arterial sistólica (PAS), pressão arterial diastólica (PAD) e pressão arterial média (PAM).

Os valores da hemogasometria arterial foram obtidos através de um analisador de gases sanguíneos (Radiometer Copenhagen ABL 555). Já as variáveis hemodinâmicas foram registradas por um transdutor de pressão conectado na artéria radial, sendo obtidas através de monitor multiparâmetro (DX 2020, Dixtal).

No ato da mensuração das variáveis de interesse, os pacientes estavam em posição supina, intubados com tubo endotraqueal e ventilados mecanicamente através do aparelho Vela (Viasys Healthcare Inc.). Os pacientes sedados entraram no protocolo do estudo durante o despertar diário de UTI. O nível de sedação foi o suficiente para que não ocorresse inibição do estímulo ventilatório, permitindo que o mesmo tivesse condições de iniciar o disparo do ventilador. No entanto, a mensuração da mecânica ventilatória estática foi obtida na ausência de drive ventilatório.

Os dados foram coletados durante três períodos: antes do suspiro (PRÉ), imediatamente após (PÓS) e 15 minutos depois da técnica (PÓS-15min). Na fase PRÉ, foi realizada a padronização e avaliação da mecânica pulmonar em um modo limitado à volume $(6 \mathrm{ml} / \mathrm{kg}$ de peso corporal predito pela altura), fluxo constante e suficiente para atender a demanda ventilatória do paciente e uma frequência respiratória ajustada para manter níveis de $\mathrm{PaCO}_{2}$ e pH dentro dos parâmetros de normalidade. Em seguida, a manobra de recrutamento alveolar por meio de suspiro foi aplicada durante uma hora.

A técnica consistiu no ajuste do ventilador mecânico no modo de ventilação mandatória intermitente sincronizada (SIMV), com uma frequência respiratória programada em duas incursões por minuto, durante um tempo inspiratório de quatro segundos, e uma pressão em vias aéreas limitada em $40 \mathrm{cmH}_{2} \mathrm{O}$. A SIMV foi associada à ventilação por pressão de suporte (PSV), ofertando um volume corrente de $6 \mathrm{ml} / \mathrm{kg}$ de peso corpóreo. Ao fim do período do suspiro, foram reavaliadas a mecânica pulmonar, os valores hemogasométricos e hemodinâmicos (mensurações PÓS). Sequencialmente, o paciente retornava ao modo PSV $(6 \mathrm{ml} / \mathrm{kg})$ e, após 15 minutos, era submetido a nova avaliação das variáveis de interesse (mensurações PÓS-15min). Durante os três períodos, a PEEP e a $\mathrm{FiO}_{2}$ não foram alteradas. 
Os dados da mecânica pulmonar e hemodinâmica foram obtidos pelo pesquisador responsável pelo estudo, exceto a amostra de sangue para mensuração dos gases sanguíneos que foi coletada pelo médico plantonista da unidade. Não houve missing data no presente estudo. Todas as variáveis de interesse foram coletadas em todas as etapas do estudo, para todos os participantes.

A análise estatística foi realizada através do software IBM SPSS versão 22.0. As variáveis numéricas de distribuição simétrica foram expressas em média aritméticas (MA) e desvio padrão (DP). Os dados categóricos foram apresentados em frequência das categorias, sendo representadas em percentagem. A análise de variância para medidas repetidas (One Way ANOVA) foi utilizada para avaliar o comportamento das variáveis no PRÉ-SUSPIRO, PÓS e PÓS-15min. Para comparar os grupos que foram estatisticamente diferentes, foi utilizado o teste post-hoc de Bonferroni. O nível de significância estatística foi estabelecido em 0,05 ou 5\%.

O projeto de pesquisa foi aprovado pelo Comitê de Ética em Pesquisa da Universidade Salvador (UNIFACS), parecer número 04.10.90, atendendo às Resoluções 466/12 e 510/16 do Conselho Nacional de Saúde.

\section{Resultados}

Durante o período de três meses em que o estudo foi realizado, 31 pacientes internados na Unidade de Cuidados Intensivos do referido hospital eram elegíveis. Destes, apenas 17 pacientes atendiam aos critérios de inclusão e exclusão, estando submetidos à VM e apresentando uma relação $\mathrm{PaO}_{2} / \mathrm{FiO}_{2}$ inferior a $300 \mathrm{mmHg}$ (figura 1). As características demográficas e clínicas dos pacientes avaliados e os principais parâmetros ventilatórios utilizados são apresentados na Tabela 1.

Figura 1. Fluxograma de elegibilidade dos pacientes na Unidade de Cuidados Intensivos (UCI) de um hospital geral-2010

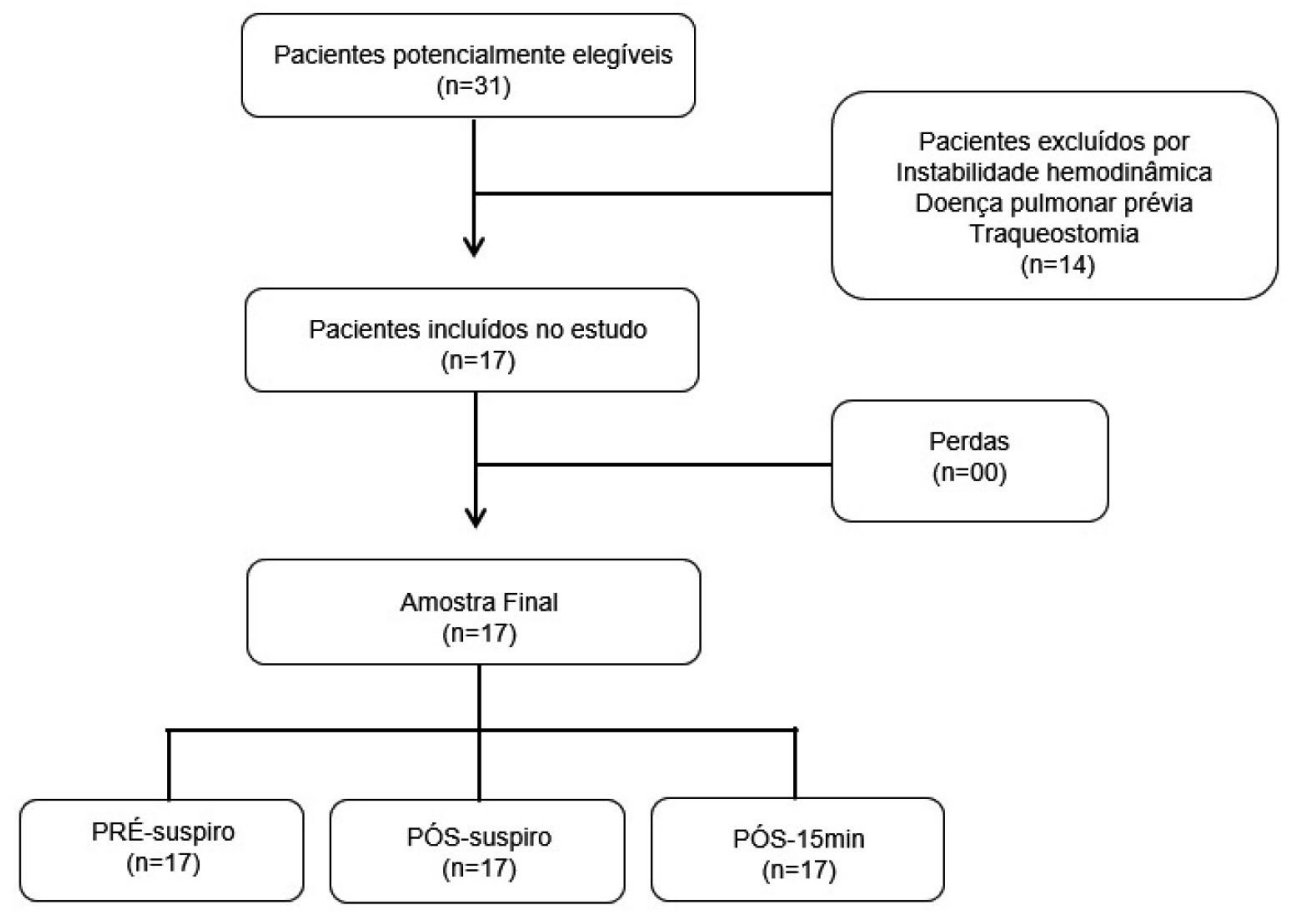


Tabela 1. Características demográficas e clínicas dos pacientes avaliados na Unidade de Cuidados Intensivos (UCI) de um hospital geral-2010

\begin{tabular}{|c|c|}
\hline Variáveis & $\begin{array}{l}\text { Amostra } \\
(n=17)\end{array}$ \\
\hline Idade (anos) & $57,7 \pm 15,8$ \\
\hline Gênero masculino, n (\%) & $10(59)$ \\
\hline $\mathrm{IMC}\left(\mathrm{Kg} / \mathrm{m}^{2}\right)$ & $22,1 \pm 0,9$ \\
\hline \multicolumn{2}{|l|}{ Diagnóstico admissional, n (\%) } \\
\hline Sepse & $7(41)$ \\
\hline Pneumonia & $6(35)$ \\
\hline Edema agudo de pulmão & $2(12)$ \\
\hline Outros & $2(12)$ \\
\hline HAS, n (\%) & $8(47)$ \\
\hline $\mathrm{DM}, \mathrm{n}(\%)$ & $10(59)$ \\
\hline Tabagismo, n (\%) & $0(0)$ \\
\hline Alcoolismo, n (\%) & $1(6)$ \\
\hline Obesidade, n (\%) & $0(0)$ \\
\hline Pneumopatia, n (\%) & $0(0)$ \\
\hline Doença cardiovascular, n (\%) & $9(53)$ \\
\hline Neuropatias, n (\%) & $0(0)$ \\
\hline Neoplasias, n (\%) & $2(12)$ \\
\hline $\operatorname{PEEP}\left(\mathrm{cmH}_{2} \mathrm{O}\right)$ & $10,3 \pm 2,5$ \\
\hline $\mathrm{PaO}_{2} / \mathrm{FiO}_{2}(\mathrm{mmHg})$ & $196,5 \pm 55,6$ \\
\hline $\mathrm{PSV}\left(\mathrm{cmH}_{2} \mathrm{O}\right)$ & $14,4 \pm 3,9$ \\
\hline
\end{tabular}

IMC: índice de massa corpórea; HAS: hipertensão arterial sistêmica; DM: diabetes mellitus; PEEP: pressão positiva no final da expiração; $\mathrm{PaO}_{2} / \mathrm{FiO}_{2}$ : relação entre pressão parcial de oxigênio alveolar e fração inspirada de oxigênio; PSV: ventilação por pressão de suporte

Ao analisar os dados observa-se que houve predominância do gênero masculino (59\%) e que a idade foi de 57,7 $\pm 15,8$ anos. Houve predomínio de sepse de foco respiratório secundário a pneumonia (35\%), sendo que todos os pacientes admitidos no estudo foram ventilados sob modo PSV, no qual o nível de pressão estabelecida foi de $14,4 \pm 3,9 \mathrm{cmH}_{2} \mathrm{O}$. O nível de PEEP utilizado foi de $10,3 \pm 2,5 \mathrm{cmH}_{2} \mathrm{O}$ e a relação $\mathrm{PaO}_{2} / \mathrm{FiO}_{2}$ foi de $196,5 \pm 55,6 \mathrm{mmHg}$ antes da instituição da manobra de recrutamento.

O comportamento da mecânica pulmonar e oxigenação nos três períodos no qual foi realizado o protocolo do estudo estão ilustrados na Tabela 2.

Tabela 2. Comportamento da mecânica pulmonar e oxigenação antes e após a aplicação do suspiro, na Unidade de Cuidados Intensivos (UCI) de um hospital geral-2010

\begin{tabular}{|c|c|c|c|}
\hline & \multicolumn{3}{|c|}{ Amostra $(n=17)$} \\
\hline & Pré & Pós & Pós-15 minutos \\
\hline$P_{\mathrm{PICO}}\left(\mathrm{cmH}_{2} \mathrm{O}\right)$ & $34,7 \pm 5,6$ & $35,5 \pm 4,5$ & $35,2 \pm 4,8$ \\
\hline P PLATô $\left(\mathrm{cmH}_{2} \mathrm{O}\right)$ & $25,5 \pm 5,3$ & $23,1 \pm 4,7^{*}$ & $23,8 \pm 4,8^{* *}$ \\
\hline $\mathrm{P}_{\mathrm{R}}\left(\mathrm{cmH}_{2} \mathrm{O}\right)$ & $9,2 \pm 2,3$ & $12,4 \pm 3,3^{\#}$ & $11,4 \pm 3,1^{*}$ \\
\hline Cest $_{\mathrm{s}, \mathrm{r}}\left(\mathrm{mL} / \mathrm{cmH}_{2} \mathrm{O}\right)$ & $36,3 \pm 11,4$ & $42,4 \pm 11,3^{\#}$ & $40,3 \pm 10,8^{¥}$ \\
\hline $\mathrm{PaO}_{2}(\mathrm{mmHg})$ & $89,6 \pm 13,0$ & $116,3 \pm 18,2^{\#}$ & $104,8 \pm 14,9^{7}$ \\
\hline $\mathrm{PaCO}_{2}(\mathrm{mmHg})$ & $35,5 \pm 6,7$ & $32,9 \pm 6,8^{*}$ & $34,3 \pm 7,3$ \\
\hline $\mathrm{PaO}_{2} / \mathrm{FiO}_{2}(\mathrm{mmHg})$ & $196,5 \pm 55,6$ & $256,4 \pm 79,9^{\#}$ & $230,3 \pm 68,6^{¥}$ \\
\hline $\operatorname{VE}(L)$ & $8,1 \pm 1,1$ & $8,8 \pm 0,9$ & $8,5 \pm 1,5$ \\
\hline
\end{tabular}

"Pós-suspiro < Pré-suspiro $(p<0,05)$; ${ }^{* *}$ Após 15minutos < Pré-suspiro $(p<0,05)$; "Pós-suspiro $>$ Pré-suspiro $(p<0,05)$; ${ }^{¥}$ Após 15 minutos $>$ Pré-suspiro $(p<0,05)$.

$\mathrm{P}_{\mathrm{PICO}}$ : pressão de pico; $\mathrm{P}_{\mathrm{PLATO}}$ : pressão de platô; $\mathrm{P}_{\mathrm{R}}$ : pressão resistiva; Cest $_{\text {s,r }}$ : complacência estática do sistema respiratório; $\mathrm{PaO}_{2}$ : pressão arterial de oxigênio; $\mathrm{PaCO}_{2}$ : pressão arterial de dióxido de carbono; $\mathrm{PaO}_{2} / \mathrm{FiO}_{2}$ : relação entre pressão parcial de oxigênio alveolar e fração inspirada de oxigênio; VE: volume minuto. 
Foi verificado que a introdução de dois suspiros por minuto com pressão em vias aéreas limitada em $40 \mathrm{cmH}_{2} \mathrm{O}$ durante quatro segundos levou a uma melhora estatisticamente significante da $\mathrm{PaO}_{2}(\mathrm{p}<0,001)$. A Cest $\mathrm{s}_{\mathrm{s}, \mathrm{r}}$ apresentou aumento estatisticamente significante logo após a retirada do suspiro $(p=0,03)$ e manteve-se estável após 15 minutos da interrupção da técnica. O comportamento das trocas gasosas durante o protocolo apresentou diferenças estatisticamente significantes quanto à aplicação da terapêutica, evidenciado pelo aumento da relação $\mathrm{PaO}_{2} / \mathrm{FiO}_{2}$ logo após a supressão do suspiro $(p<0,001)$ e esse incremento foi mantido durante os 15 minutos restantes $(p<0,001)$.

O comportamento hemodinâmico durante a realização do protocolo está ilustrado na Tabela 3.

Tabela 3. Comportamento hemodinâmico antes e após a aplicação do suspiro na Unidade de Cuidados Intensivos (UCI) de um hospital geral,2010

\begin{tabular}{llll}
\hline \multirow{2}{*}{ Variáveis } & \multicolumn{3}{c}{ Amostra $(\mathbf{n}=17)$} \\
\cline { 2 - 4 } & Pré & Pós & Pós-15 minutos \\
\hline FC $(\mathrm{bpm})$ & $96,0 \pm 16,0$ & $92,7 \pm 14,6$ & $96,4 \pm 13,1$ \\
PAS $(\mathrm{mmHg})$ & $128,2 \pm 17,5$ & $130,2 \pm 12,0$ & $128,5 \pm 15,1$ \\
PAD $(\mathrm{mmHg})$ & $68,2 \pm 12,1$ & $70,2 \pm 10,7$ & $69,5 \pm 12,2$ \\
PAM (mmH) & $87,8 \pm 12,5$ & $89,6 \pm 9,7$ & $88,7 \pm 11,3$ \\
\hline F: frequência cardíaca; PAS: pressão arterial sistólica; PAD: pressão arterial diastólica; PAM: pressão arterial média.
\end{tabular}

As variáveis hemodinâmicas não apresentaram diferenças estatisticamente significantes com a aplicação da manobra de recrutamento através de suspiro, mantendo-se estabilizadas quando passados 15 minutos da aplicação da técnica.

Não foram registradas intercorrências associadas à utilização da técnica de suspiro no que diz respeito a desenvolvimento de instabilidade hemodinâmica durante aplicação do protocolo, caracterizado pela PAM < 60mmHg; depressão do drive respiratório, ou exteriorização de secreção traqueal.

\section{Discussão}

A adição de dois suspiros intermitentes por minuto em associação com o modo PSV melhorou a oxigenação arterial e a Cest ${ }_{s, r}$ e, possivelmente, promoveu o recrutamento alveolar de pacientes com relação $\mathrm{PaO}_{2} / \mathrm{FiO}_{2}<300$ submetidos à VM.

Os efeitos positivos da manobra de recrutamento alveolar sobre as trocas gasosas podem estar associados com o incremento no volume corrente, sugerindo que a aplicação de suspiro possa prevenir o colapso alveolar em pacientes que apresentem déficit nas trocas gasosas. É válido salientar que as manobras de recrutamento alveolar devem ser usadas no contexto de proteção pulmonar e não apenas como meio de melhorar a oxigenação 7 .

Outro parâmetro é o tempo de sustentação ou manutenção da pressão média em vias aéreas que pode favorecer o preenchimento alveolar, equilibrando as constantes de tempo do sistema respiratório e, consequentemente, melhorando a distribuição gasosa.

Em consonância a isto, Güldner et al. .6 , em seu estudo experimental com suínos recebendo $60 \%$ do volume corrente em suspiros, observaram uma melhora na oxigenação pulmonar com redistribuição de perfusão que implicou na diminuição do gradiente ventilação/perfusão, sem alterações no peso pulmonar (por formação de edema ou carga inflamatória). Estes resultados foram confirmados por Mauri et al. $\frac{13}{}$ relatando melhora da $\mathrm{PaO}_{2}$ e diminuição do volume corrente regional, ou seja, o suspiro proporcionou uma melhor distribuição regional deste volume favorecendo uma diminuição da heterogeneidade pulmonar com redução da sua distensão. 
Em termos de viscoelasticidade pulmonar, Antonaglia et al. ${ }^{12}$ evidenciaram uma forte relação da melhora da $\mathrm{PaO}_{2}$ com estas propriedades, indicando que quanto maior a variação viscoelástica, maior o potencial de ganho em termos de recrutamento de áreas colapsadas por redução do gradiente pulmonar heterogêneo. Contudo, devido à variação de pacientes com propriedades viscoelásticas diferentes e a quantidade amostral reduzida, não foram evidenciadas alterações significativas em termos de $\mathrm{PaO}_{2}$ e Cest ${ }_{\mathrm{s}, \mathrm{r}}{ }^{12}$.

Neste aspecto, em um ensaio clínico randomizado realizado por Mauri, et al. $\frac{17}{}$, que comparou o modo Ventilação por Pressão de Suporte (PSV) e PSV associado a suspiro, não foram encontradas diferenças expressivas em termos de relação $\mathrm{PaO}_{2} / \mathrm{FiO}_{2}$ e desfechos clínicos importantes como: tempo de permanência hospitalar, dias de ventilação mecânica e mortalidade. Porém, foi demonstrado ser uma manobra segura, sem eventos adversos e sem danos teciduais.

Steimback et al. $\frac{18}{\frac{1}{2}}$ identificaram resultados positivos, principalmente em termo de dano tecidual. Contudo, a adição de 3 suspiros por minuto pareceu ser lesiva quando comparada a 1 suspiro a cada 6 minutos. Sendo assim, esses autores sugerem que, em ratificação ao estudo prévio de Vaporidi et al. $\stackrel{19}{ }$, a frequência de exposição do suspiro pode estar relacionada com a capacidade reparadora intrínseca do epitélio pulmonar.

Analisando a sustentação dos efeitos alcançados pela manobra de recrutamento alveolar através de suspiro, Pelosi et al. $\frac{16}{6}$ evidenciaram que, após a interrupção desta, todas as variáveis de mecânica do sistema respiratório retornaram aos valores basais. Já Rival et al. $\underline{20}$, ao submeterem pacientes com SDRA a suspiros prolongados de $45 \mathrm{CmH}_{2} \mathrm{O}$ associados à posição prona, demonstraram melhora acentuada da oxigenação, mantendo o aumento adquirido na relação $\mathrm{PaO}_{2} / \mathrm{FiO}_{2}$ por até 13 horas após o final do estudo.

Logo após a suspensão do suspiro e passados $15 \mathrm{mi}-$ nutos desta suspensão, os valores da Cest ${ }_{s, r^{\prime}} \mathrm{PaO}_{2}$ e as trocas gasosas apresentaram diferenças estatisticamente significantes, indicando que as propriedades viscoelásticas ou a heterogeneidade do sistema respiratório, juntamente com a oxigenação, podem ser melhoradas com o suspiro. Contudo, é importante ressaltar que estes valores são dependentes da elastância pulmonar, tendo sido evidenciada uma tendência na redução de seus valores, sugerindo que a persistência do suspiro por um tempo maior possa ser benéfica na manutenção destes valores.

Outra variável que também apresentou elevação durante a aplicação da técnica e manteve-se após 15 minutos do suspiro foi a PR. Esta elevação pode ser explicada através da interação de volumes correntes elevados e o fluxo, tendo como produto final a resistência elevada devido a passagem turbulenta do ar. A manutenção desses valores posterior à conduta pode ser explicada pela reatividade da parede brônquica a fluxos elevados ${ }^{7}$. Considerando o comportamento da PR, a revisão da literatura demonstrou que este foi, até o presente momento, o único estudo a avaliar esta variável, podendo, portanto, ser considerado como pioneiro.

As manobras de recrutamento não são isentas de ris$\cos ^{5}$. Estudos sugerem que lesões pulmonares como barotrauma, surgimento de pneumotórax, arritmias, hipotensão arterial e a dessaturação são os eventos adversos que podem ocorrer, sendo os dois últimos mais comuns durante as manobras de recrutamento ${ }^{5,21}$. As variáveis hemodinâmicas analisadas neste estudo não apresentaram diferenças estatisticamente significantes, mantendo-se estabilizadas, durante e após a manobra de recrutamento, ou seja, nenhum dos pacientes em questão apresentou instabilidade hemodinâmica. Além disso, não foram registrados episódios de dessaturação durante a aplicação do protocolo. Resultados semelhantes foram encontrados por Patroniti et al. ${ }^{11}$ ao demonstrar que, durante a administração da manobra de recrutamento com suspiros intermitentes, os parâmetros hemodinâmicos não sofreram quaisquer alterações significativas ao longo do estudo. Neste aspecto, Mauri et al. ${ }^{17}$ também não identificaram alterações hemodinâmicas, presença de barotraumas e arritmias significativas no grupo PSV com Suspiro versus grupo PSV, demonstrando ser uma manobra segura e possível de ser realizada sem prejuízos clínicos.

Apesar dos resultados apresentados serem considerados substanciais e com significado clínico, ainda se faz necessário a realização de ensaios clínicos, randomizados, controlados, na tentativa de elucidar qual é a estratégia de recrutamento alveolar mais adequada para incremento da mecânica pulmonar e oxigenação em pacientes submetidos à ventilação mecânica. 
Dentre as limitações desse estudo, tem-se a utilização de uma amostragem não probabilística, por conveniência, reduzindo a capacidade de generalização dos resultados com precisão estatística. Além disso, não é possível assegurar a segurança da técnica visto que os pacientes foram acompanhados apenas por 15 minutos após a realização do suspiro. O que se pode afirmar é que durante o período de realização do suspiro e após 15 minutos da sua realização, não foram registrados impacto hemodinâmico e intercorrências associadas à utilização da técnica.

\section{Conclusão}

Neste estudo, a utilização de suspiros em pacientes submetidos à mecânica invasiva foi capaz de melhorar a oxigenação e a mecânica pulmonar, sem comprometer a estabilidade hemodinâmica. A técnica pode, portanto, ser considerada como uma estratégia eficaz para manutenção do recrutamento alveolar e prevenção de possíveis atelectasias.

\section{Contribuições dos autores}

Batista Neto AF, Avena KM e Mendes KMB contribuíram na concepção, desenvolvimento e redação do artigo científico, tendo aprovado a versão final encaminhada para publicação. Duarte HB contribuiu na redação do artigo científico, tendo aprovado a versão final encaminhada para publicação. Olivieri FM e Feijó LF contribuíram no desenvolvimento e redação do artigo científico, tendo aprovado a versão final encaminhada para publicação.

\section{Conflitos de interesses}

Nenhum conflito financeiro, legal ou político envolvendo terceiros (governo, empresas e fundações privadas, etc.) foi declarado para nenhum aspecto do trabalho submetido (incluindo, mas não se limitando a subvenções e financiamentos, participação em conselho consultivo, desenho de estudo, preparação de manuscrito, análise estatística, etc.).

\section{Referências}

1. Gattinoni L, Quintel M, Marini JJ. “Less is More” in mechanical ventilation. Intensive Care Med. 2020;46(4):780-2. https://doi. org/10.1007/s00134-020-05981-z

2. Ware LB. Pathophysiology of acute lung injury and the acute respiratory distress syndrome. Semin Respir Crit Care Med. 2006;27(4):337-49. https://doi.org/10.1055/s-2006-948288

3. Suzumura EA, Figueiró M, Normilio-Silva K, Laranjeira L, Oliveira C, Buehler AM, et al. Effects of alveolar recruitment maneuvers on clinical outcomes in patients with acute respiratory distress syndrome: a systematic review and meta-analysis. Intensive Care Med. 2014;40(9):1227-40. https://doi.org/10.1007/s00134-0143413-6

4. Barbas CS, Ísola AM, Farias AM, Cavalcanti AB, Gama AM, Duarte AC. Recomendações brasileiras de ventilação mecânica 2013. Parte I. Rev bras ter Intensiva. 2014;26(2):89-121. http:// dx.doi.org/10.5935/0103-507X.20140017

5. Cavalcanti AB, Suzumura ÉA, Laranjeira LN, Paisani DM, Damiani LP, Guimarães HP, et al. Effect of lung recruitment and titrated positive end-expiratory pressure (PEEP) vs low PEEP on mortality in patients with acute respiratory distress syndrome: a randomized clinical trial. JAMA. 2017;318(14):1335-45. https://doi. org/10.1001/jama.2017.14171

6. Güldner A, Braune A, Carvalho N, Beda A, Zeidler S, Wiedemann $B$, et al. Higher levels of spontaneous breathing induce lung recruitment and reduce global stress/strain in experimental lung injury. Anesthesiology. 2014;120(3):673-82. https://doi. org/10.1097/aln.0000000000000124

7. Hess DR. Recruitment Maneuvers and PEEP Titration. Respir Care. 2015;60(11):1688-1704. https://doi.org/10.4187/ respcare.04409

8. Hartland BL, Newell TJ, Damico N. Alveolar recruitment maneuvers under general anesthesia: a systematic review of the literature. Respir Care. 2015;60(4):609-20. https://doi.org/10.4187/ respcare.03488

9. Santos CL, Samary CS, Fiorio Júnior PL, Santos BL, Schanaider A. Recrutamento pulmonar na síndrome do desconforto respiratório agudo. Qual a melhor estratégia?. Rev Col Bras Cir. 2015;42(2):125-9. https://doi.org/10.1590/0100-69912015002010 
10. Antonaglia V, Pascotto S, Simoni LD, Zin WA. Effects of a sigh on the respiratory mechanical properties in ALI patients. J Clin Monit Comput. 2006;20(4):243-9. https://doi.org/10.1007/s10877006-9028-X

11. Patroniti N, Foti G, Cortinovis B, Maggioni E, Bigatello LM, Cereda $\mathrm{M}$, et al. Sigh improves gas exchange and lung volume in patients with acute respiratory distress syndrome undergoing pressure support ventilation. Anesthesiology. 2002;96(4):788-94. https://doi.org/10.1097/00000542-200204000-00004

12. Antonaglia V, Peratoner A, De Simoni L, Lucangelo V, Gullo A, Zin WA. Sigh: tool to determine the respiratory viscoelastic properties. J Clin Monit Comput. 2002;17(7-8):459-66. https://doi. org/10.1023/A:1026277004613

13. Moraes L, Santos CL, Santos RS, Cruz FF, Saddy F, Morales MM, et al. Effects of sigh during pressure control and pressure support ventilation in pulmonary and extrapulmonary mild acute lung injury. Crit Care. 2014;18(4):474. https://doi.org/10.1186/s13054014-0474-4

14. Mauri T, Eronia N, Abbruzzese C, Marcolin R, Coppadoro $A$, Spadaro S, et al. Effects of sigh on regional lung strain and ventilation heterogeneity in acute respiratory failure patients undergoing assisted mechanical ventilation. Crit Care Med. 2015;43(9):1823-31. https://doi.org/10.1097/ ccm.0000000000001083

15. Tabuchi A, Nickles HT, Kim M, Semple JW, Koch E, Brochard L, et al. Acute lung injury causes asynchronous alveolar ventilation that can be corrected by individual sighs. Am J Respir Crit Care Med. 2016;193(4):396-406. https://doi.org/10.1164/rccm.201505$\underline{09010 \mathrm{C}}$
16. Pelosi P, Cadringher P, Bottino N, Panigada M, Carrieri F, Riva $E$, et al. Sigh in acute respiratory distress syndrome. Am J Respir Crit Care Med. 1999;159(3):872-80. https://doi.org/10.1164/ ajrccm.159.3.9802090

17. Mauri T, Foti G, Fornari C, Grasselli G, Pinciroli R, Lovisari F, et al. Sigh in patients with acute hypoxemic respiratory failure and acute respiratory distress syndrome: the PROTECTION pilot randomized clinical trial. Chest. 2020. https://doi.org/10.1016/j. chest.2020.10.079

18. Steimback PW, Oliveira GP, Rzezinski AF, Silva PL, Garcia CS, Rangel $G$, et al. Effects of frequency and inspiratory plateau pressure during recruitment manoeuvres on lung and distal organs in acute lung injury. Intensive Care Med. 2009;35(6):1120 8. https://doi.org/10.1007/s00134-009-1439-y

19. Vaporidi K, Voloudakis G, Priniannakis G, Kondili E, Koutsopoulos A, Tsatsanis C, et al. Effects of respiratory rate on ventilator-induced lung injury at a constant $\mathrm{PaCO} 2$ in a mouse model of normal lung. Crit Care Med. 2008; 36(4):1277-83. https:// doi.org/10.1097/ccm.0b013e318169f30e

20. Rival G, Patry C, Floret N, Navellou JC, Belle E, Capellier G. Prone position and recruitment manoeuvre: the combined effect improves oxygenation. Crit Care. 2011;15(3):R125. https://dx.doi. org/10.1186\%2Fcc10235

21. Fan E, Wilcox ME, Brower RG, Stewart TE, Mehta S, Lapinsky SE, et al. Recruitment maneuvers for acute lung injury: a systematic review. Am J Respir Crit Care Med. 2008;178(11):1156-63. https:// doi.org/10.1164/rccm.200802-3350C 